\title{
CANDIDA AuRIS: EMERgenCe AND EPIDEMIOLOgY Of A HIGHLY
} PATHOGENIC YEAST

\author{
Paula Slomp Santos ${ }^{1}$, Daiane Flores Dalla Lana², Adelina Mezzari
}

\begin{abstract}
Candida auris is a multidrug-resistant emerging yeast, which was responsible for healthcare-associated infection outbreaks, and was cataloged as a new species in 2009 , after being isolated from a patient's ear canal secretion in Japan. Since the notification of this first occurrence, numerous cases have been reported throughout the world, including Brazil. C. auris affects mainly inpatients, patients in intensive care units, exposed to broad-spectrum antifungal medications and who make use of vascular catheters. Currently, this yeast is one of the main responsible for invasive infections in hospitals and has been cause of concern by authorities and organs due to its rapid dissemination and difficult treatment caused by its low susceptibility to antifungal agents traditionally used in clinical practice. As a contributor to the severity of infections associated with $C$. auris, the transmission mechanism is still unknown, which implies in a lack of control of the microorganism and high mortality rates. Thus, this literature review presents relevant information in order to alert the importance of $C$. auris as an etiological agent of systemic infections, as well as its epidemiology and the real challenges of the treatment.
\end{abstract}

Keywords: Candida auris; candidiasis; candidemia; multidrug-resistance; biofilm; epidemiology; diagnosis

Infections caused by Candida genus yeasts are known as candidiasis or candidosis ${ }^{1}$. Candidiasis, among other clinical forms, can also be considered a sexually transmitted disease (SDT) and is clinically manifested through lesions, which can be classified as superficial with cutaneous and mucosal involvement, or systemic, disseminated, and of high severity ${ }^{2}$. This type of mycosis has the mouth, the throat, the tongue, scalp, genitals, fingers, nails and internal organs as infection sites ${ }^{1}$. Its origin can be exogenous or endogenous, being the latter the main transmission mechanism, in which the Candida species that are part of the microbiota in tissues and organs, when faced with the vulnerability of the host, for example in immunocompromised situations, become opportunistic. The exogenous way is a transmission mechanism that occurs mainly through direct contact of health professionals with patients or contaminated medical-hospital issues ${ }^{2}$.

The main virulence factors of yeasts that induce infection are $^{3}$ :

a) secretion of extracellular enzymes such as phospholipases and proteinases, which degrade the host tissue leading to tissue invasion ${ }^{3}$;

b) Production of toxic substances that damage the cells ${ }^{3}$;

c) Creation of biofilms on cells and inert surfaces ${ }^{3}$;

d) Formation of pseudohyphae by certain species of Candida spp.3;

e) Production of hemolysins ${ }^{2}$;

f) The ability to adhere to medical and hospital materials and host cells ${ }^{3}$.

The sum of these mechanisms with the weakness of the host response may lead to candidiasis ${ }^{3}$.

Microorganisms usually live naturally in communities, through the formation of biofilm; this is due to the advantages of being in association, among which
Clin Biomed Res. 2017;37(3):247-254

1 Department of Analyses, School of Pharmacy, Universidade Federal do Rio Grande do Sul (UFRGS). Porto Alegre, RS, Brazil.

2 Graduate Program in Pharmaceutical Sciences, School of Pharmacy, Universidade Federal do Rio Grande do Sul (UFRGS). Porto Alegre, RS, Brazil.

Corresponding author:

Adelina Mezzari

mezzari@ufrgs.br

Departamento de Análises, Faculdade de Farmácia, Universidade Federal do Rio

Grande do Sul (UFRGS)

Av. Ipiranga, 2752.

90610-000, Porto Alegre, RS, Brazil. 
the main one is a greater protection against the action of antifungal agents and the immune system defenses of the host ${ }^{2}$.

The epidemiology of Candida spp. can be variable, according to the virulence of the isolate, parasitic load and predisposition of the host, and all these factors make them pathogenic yeasts ${ }^{1}$. Candida species are responsible for bloodstream infections (BSIs) in hospitalized patients, and affect mainly those who are in intensive care units, exposed to broad spectrum antifungal agents, internal vascular catheters, parenteral nutrition, abdominal surgery and immunosuppressants ${ }^{4}$. The BSIs represent a challenge to public health due to their severity and intensity, leading to an increase in the length of hospital stay, increasing hospitalization costs, as well as morbidity and mortality rates in infected patients. In studies conducted throughout 3 years in 49 hospitals in the United States, Candida spp. was indicated as one of the main etiological agents of BSIs, responsible for $7.6 \%$ of the cases ${ }^{2}$.

Among the most common causes of BSIs is candidemia, which is the syndrome most frequently associated with invasive candidiasis ${ }^{5}$ and high mortality rates $(40 \% \text { to } 60 \%)^{2}$. Invasive candidiasis is one of the major clinical manifestations of the Candida genus caused by $C$. albicans and non-albicans ${ }^{6}$ Candida, these, in turn, respond to at least $50 \%$ of the invasive infections by Candida spp. ${ }^{3}$, and can be fluconazole-resistant or difficult to extinguish due to the fact that they produce biofilm ${ }^{7}$. The Candida yeast has more than 200 existing species, among which are exemplified especially - C. albicans, C. glabrata, C. parapsilosis, C. krusei, C. tropicalis, C. haemulonii, C. guilliermondii, and the most recently identified species, C. auris ${ }^{1,6}$. The latter has been classified as a highly pathogenic new species and it is multiresistant to antifungal agents traditionally used in clinical practice, being the cause of several systemic infections, which can be fatal ${ }^{6}$. In 2013, a study reported $33 \%$ of lethality in cases of BSIs attributed to $C$. auris in all patients and $57 \%$ in the subgroup of patients admitted to intensive care therapy units; however these rates can be attributed to the severity of underlying diseases in these patients ${ }^{8}$.

The rapid emergence of $C$. auris and the resistance to the three major classes of antifungal drugs (azoles, echinocandins and polyenes) ${ }^{9}$, the horizontal transmission among hospitalized patients, leading to healthcare-associated infection outbreaks, and the high mortality rates associated, make $C$. auris one of the most current causes of invasive infections in hospitals and reason of concern due to its evolution and worldwide spread ${ }^{4}$. Furthermore, fungal otomastoiditis, which is a rare and possibly fatal disease for immunosuppressed patients, has C. auris ${ }^{10,11}$ as its etiologic agent. There have been increasing cases of otologic infection caused by C. auris ${ }^{10}$. Although the origin of the infections is inconclusive, it is not yet known whether these Isolates are in the hospital environment or are of endogenous origin ${ }^{12}$. Evidence indicates that the dissemination of C. auris in the hospital environment may occur through contact with contaminated surfaces and devices and also in an interpersonal way, thus alerting services to this opportunistic pathogen ${ }^{9}$.

Given the above, this review aims to address epidemiological aspects of $C$. auris candidiasis, evidencing cases already described in Brazil as well as frequency of cases by region. Additionally, we present the methods most used for the identification of $C$. auris, the data of microbial susceptibility against the most varied classes of antifungal drugs, and therapeutic challenges. Therefore, we intend to contribute to the elucidation of the clinical importance of the theme, gathering relevant information for a better understanding of which are the factors that promote the expansion and opportunism of the disease, which generates an alert about this noticeable species among the non-albicans Candida species currently, in regard to high virulence and pathogenicity.

\section{METHODS}

A systematic bibliographic review study was conducted in databases and online collections of the Elsevier Publisher (ScienceDirect) and the National Institutes of Health (PubMed) virtual health library from April to June 2017.

The terms used in the electronic searches were "Candida auris", "candidíase/candidiasis", "candidemia", "Candida auris diagnóstico/diagnosis", "Candida auris epidemiologia/epidemiology", "Candida auris multirresistente/multidrug-resistant", "Candida auris biofilme/biofilm" and "Candida auris tratamento/ treatment".

In addition, an online survey was conducted to access documents of national (ANVISA) and international organizations, which could contribute with data to compose the present study.

The purpose of this research was to address the most relevant and current aspects of identification, epidemiology, clinical manifestations, treatment, and prevention of infections by $C$. auris, a species still little known in Brazil, whose information is still controversial between official records and scholars, indicating the demand for care and attention.

The inclusion criteria were scientific papers that explored the proposed theme, published between the 2003 and 2017. We excluded studies that did 
not have adequate bibliographic references, which were incomplete or tertiary sources. In addition, it we decided not to include theses, dissertations, monographs and books.

\section{Identification}

In order for the dissemination of $C$. auris to be prevented and controlled in a hospital environment, it is of the utmost importance that the identification of the isolate be done quickly. When the identification of the species is suspected in an isolate, or even after confirmation, it is the responsibility of the laboratory to immediately inform the Hospital Infection Control Committee $(\mathrm{CClH})$ of the health service ${ }^{9}$.

Infections caused by this new species of Candida are diagnosed by blood culture or culture of other body fluids. Its specific identification requires molecular laboratory methods, such as D1-D2 or Internal Transcribed Spacer (ITS) $)^{9,12,13}$, or desorption/ionization with mass spectrometry, and the Matrix-Assisted Laser Desorption lonization - Time of Flight (MALDI-TOF), the fastest and most suitable method described in the literature for the identification of $C$. auris ${ }^{14}$, making it possible to create reference spectrum and the care to be taken when adopting this approach.

The laboratory that isolates by the phonotypical methods one of the following species: C. haemulonii, C. famata, C. sake, C. catenulata, C. Iusitaniae/C. guilliermondii, Saccharomyces cerevisiae and Rhodotorula glutinis and has the MALDI-TOF equipment or the genome sequencing should confirm whether the isolate is C. auris. It is imperative that it is made sure that MALDI-TOF contains protein profiles in its database that enable the detection of $C$. auris isolates. Currently, in Brazil, only Research Use Only (RUO) Bruker libraries of reference spectra have profiles of $C$. auris ${ }^{9}$.

In the case of laboratories that do not have the equipment, but are able to carry out screening tests for $C$. auris, they should conduct a culture examination and direct research with India ink to discard the presence of the encapsulated yeast. Subsequently, screening tests for possible identification of $C$. auris should be carried out with the aim of discarding the other non-albicans Candida species, among them filamentation tests on Tween-80 corn meal agar and/or colony color analysis on Sabouraud dextrose agar or other agar and/or color analysis on chromogenic agar and/or presence of germ tube (PGT). Isolates that do not present carotenoid pigments, negative PGT, and/or pseudohyphae formation on corn meal agar, and pink to purple coloration on chromogenic agar should be separated for further confirmation and, if positive for $C$. auris, the Commission for Hospital Infection Control (CHIC) should be notified ${ }^{9}$.
In the case of positive screening tests, the competent organ should perform biochemical tests to screen species phenotypically similar to $C$. auris; determine the susceptibility of the yeast to antifungal agents, to verify the resistance of the suspected isolate; to perform sequencing of the D1-D2 or ITS1-ITS4 regions and/or MALDI-TOF for confirmation of $C$. auris, or refer directly to reference laboratories ${ }^{9}$.

Therefore, criteria for considering an isolate suspect of $C$. auris are those phenotypically identified as C. haemulonii, C. famata, C. sake, C. catenulata, C. Iusitaniae/C. guilliermondii, $S$. cerevisiae and $R$. glutinis and that presented high minimal inhibitory concentrations (MIC) for fluconazole (FCZ; MIC $\geq 32 \mu \mathrm{g} / \mathrm{mL}$ ), voriconazole (VCZ; MIC $\geq 2 \mu \mathrm{g} / \mathrm{mL}$ ), amphotericin B (APHB; $M I C \geq 2 \mu \mathrm{g} / \mathrm{mL}$ ), and echinocandins (ECHs), such as anidulafungin (ADA; $\geq 2 \mu \mathrm{g} / \mathrm{mL}$ ) .

This new species can be erroneously identified as those mentioned above ${ }^{9,14}$, if identification is made through classical methods ${ }^{6,9}$. Gaitan et al. ${ }^{6}$ initially described isolates identified as $S$. cerevisiae, C. sake, C. Iusitaniae, C. haemulonii, and eight inconclusive isolates, and 8 (eight) were isolated from the blood and 4 (four) from the tip of the catheter, from patients hospitalized in an ICU in a European hospital. Confirmation of $C$. auris from all isolates was conducted by molecular methods. ${ }^{6}$

In Colombia, isolates were initially identified as C. haemulonii, C. famata, C. albicans or C. tropicalis. After observing the unusual prevalence and the micromorphological discordance, the strains were cultured in CHROMagar Candida medium using MALDI-TOF. Pink colonies were observed in the CHROMagar Candida medium and there was subsequently molecular confirmation of $C$. auris in all isolates ${ }^{14}$.

According to Chowdhary et al., growth at $40{ }^{\circ} \mathrm{C}$ may differentiate the isolates of $C$. auris that are mistakenly identified as $C$. haemulonii by VITEK, considering that the species Candida haemulonii does not grow at $40^{\circ} \mathrm{C} .{ }^{8,12}$ Kumar et al. reported a rapid and inexpensive method using the CHROMagar Candida supplemented with Pal's medium to differentiate $C$. auris from isolates identified as $C$. haemulonii by VITEK2. All isolates of $C$. auris showed smooth, white-to-cream colonies at $37^{\circ} \mathrm{C}$ and at $42^{\circ} \mathrm{C}$, after 24 and 48 hours of incubation and did not produce pseudohyphae. The isolates of $C$. haemulonii had a weak growth of smooth, light-pink colonies, in 24 and 48 hours, and there was no pseudohyphae production either. The $C$. haemulonii yeast did not grow at $42{ }^{\circ} \mathrm{C}^{8,15}$. 
Thus, it is observed in the literature that the identification and the diagnoses of $C$. auris are definitely carried out by molecular methods $\mathrm{s}^{6,8,9,12-21}$.

Routine methods (direct, culture, biochemical tests) are used only as screening for initial detection of yeast in clinical samples.

As a recommendation to hospitals that are not able to routinely detect this new Candida species, it is advisable to monitor the monthly number of positive blood cultures with non-albicans Candida species, as any increase may be indicative of a potential outbreak, possibly related to $C$. auris ${ }^{8}$.

\section{Epidemiology}

Due to the severity of the outbreaks that it has been causing in hospitals around the planet, $C$. auris shows a real and eminent health risk and should be observed with great caution. It was isolated for the first time from the external auditory canal of a patient in Japan and was described as a new species in $2009^{16}$. Since that episode, several other countries have reported infections, including Japan, South Korea, India, Pakistan, South Africa, Kenya, Kuwait, Brazil, Israel, Venezuela, Colombia, United Kingdom, United States, and Canada. These findings prove that the species is not limited to a particular region ${ }^{9,12}$.

The epidemiology of $C$. auris specifically in Brazil is still inconclusive. While studies conducted in $2016^{12,17,18}$ already indicate the presence of the yeast in the country, $\mathrm{ANVISA}^{9}$, through an official statement, reported that up to the present moment there have been no occurrences of infections by C. auris in our country ${ }^{9}$. However, this declaration does not exclude the possibility that the species is already present in the region, since its prevalence is not sufficiently known due to difficulties in its identification and diagnosis ${ }^{9}$.

In America, the first outbreak occurred in Venezuela between March 2012 and July 2013, in 18 affected patients, among which 13 were pediatric. The isolates were initially identified as $C$. haemulonii and after sequencing of the ITS region and analysis by Amplified Fragment Length Polymorphism (AFLP) it was identified that the microorganism involved was $C$. auris ${ }^{9}$.

Between February and July 2016 in Colombia, the outbreak occurred in 6 different hospitals totaling 17 inpatients. Among the 17 patients, 9 were male; the age group ranged between 0-77 years, 15 were hospitalized in ICUs and 2 in medical units. Of the total, 13 patients showed fungemia and in the other four, C. auris was isolated from peritoneal fluid, cerebrospinal fluid, bone, or urine. Most of the patients had central venous catheter, urinary catheter, and mechanical ventilation. Some had risk factors for candidemia: red blood cell transfusion, parenteral nutrition, abdominal surgery, hemodialysis, diabetes, pancreatitis, cancer, and HIV infection ${ }^{14}$.

In India, the highest incidence of $C$. auris infection has occurred in public hospitals. In 27 ICUs 1400 cases of Candida infection were reported, among which 74 (5.3\%) were isolated and confirmed as $C$. auris. In patients with diagnosis of candidemia, those infected with $C$. auris had an average period of hospital stay of 25 days, which was higher than those infected with non-auris Candida, with a mean of 15 days ${ }^{22}$. In the city of New Delhi, in two hospitals, 12 C. auris were isolated, collected between 2009 and 2011. The isolates from these hospitals were clonal, indicating an inter-hospital transmission. Most patients presented permanent urinary catheter and persistent candidemia - and the mortality rate was of $33 \%{ }^{23}$.

Between October 2012 and October 2013, 4 isolates were sent to the National Institute of Communicable Diseases in Johannesburg, South Africa, from 4 patients presenting candidemia and hospitalized in different institutions. The isolates were initially identified as $C$. haemulonii and $R$. glutinis by tests commonly used and later $C$. auris was correctly identified by genome sequencing ${ }^{24}$.

The first and largest outbreak of $C$. auris in Europe occurred in a cardiac center in London between April 2015 and July 2016, totaling 50 cases. Among these, $44 \%(n=22 / 50)$ developed C. auris candidemia. Through an environmental sampling the persistent presence of the yeast was detected around the spaces between the beds ${ }^{25}$.

In Spain, between April and June 2016, eight isolates from four patients (two per patient), at the Hospital Universitário e Politécnico La Fe in Valencia, were obtained from blood cultures and catheter tips. All patients were adults and were hospitalized in the ICU. The four cases were identified as $C$. auris after confirmation by sequencing the ITS region ${ }^{6}$.

Lockhart et al. ${ }^{26}$, carried out a study throughout 3 years (2012 to 2015), to understand the emergence and epidemiology of $C$. auris from isolates of 54 patients from Pakistan, India, South Africa, Venezuela and Japan. From 41 isolates, which had information regarding the patients, antifungal susceptibility testing and complete genome sequencing (WGS) testing were performed. The clinical condition of patients were $41 \%$ diabetes mellitus, $51 \%$ had undergone surgery recently, $73 \%$ had central venous catheter, and $41 \%$ were receiving systemic antifungal therapy, since $C$. auris had been isolated. The mean time since admission until infection was of 19 days, where $61 \%$ of patients had blood infection and $59 \%$ died. Among the isolates, $93 \%$ were fluconazole-resistant, $35 \%$ were resistant to amphotericin B and $7 \%$ to 
echinocandins, $41 \%$ were resistant to 2 antifungal classes and $4 \%$ to 3 classes $^{26}$.

Also, isolated cases and outbreaks have been reported in five different continents, including Europe, Asia, North America, South America, and Africa. A case in Norway, probably from another country outside the continent, confirms that there is a risk of transmission of yeast through the hospital transfer of patients. In a recent study, $C$. auris isolates present in the United Kingdom were shown to have diverse geographic origins, suggesting multiple introductions in the country ${ }^{8}$.

Considering the above, it was observed that C. auris is a species of high prevalence in various regions and climatic conditions around the world, being associated with high mortality rates in hospitalized patients. Due to the inherent difficulties of detection and diagnosis, its epidemiology is still controversial in Brazil.

\section{Susceptibility and treatment challenges}

The indiscriminate use of antifungals may have induced $C$. auris to become a multidrug-resistant pathogen, limiting treatment efficacy because of its resistance to fluconazole (FCZ), and variable susceptibility to other azoles, amphotericin B (APHB) and echinocandins $(\mathrm{ECHs})^{12,27,28}$, as it can be observed in Table $1^{21}$.

The ability to produce biofilms, the survival and dissemination in hospital settings, besides the risk of outbreaks, contribute to the pathogenicity of $C$. auris. According to the literature, $C$. auris may be as pathogenic as $C$. albicans. Despite the fact that it forms less biofilm when compared to $C$. albicans, this new species has demonstrated a greater virulence, but still needs to be further investigated. These factors, together with the resistance of $C$. auris to most antifungal agents, may explain why it is considered one of the most risky pathogens currently ${ }^{13}$.
According to Sherry et al. ${ }^{13}$, C. albicans presented a higher biofilm mass, fact that is directly related to its pathogenicity. C. auris, in turn, produced significantly less biofilm. However, the two species had a higher biofilm production compared to C. glabrata. The C. albicans biofilms were densely packed with pseudohyphae, C. glabrata formed a sparse biofilm with yeast cells, without extracellular matrix, and $C$. auris presented biofilm containing budding yeast and pseudohyphae. Still according to Sherry et al. ${ }^{13}$, broth microdilution susceptibility testing was also performed with FCZ, voriconazole (VCZ), caspofungin (CPA), micafungin (MCA), amphotericin B liposomal (APHB lipo), APHB, and chlorhexidine (CXA). For planktonic and sessile cells of $C$. auris showing MIC $>32 \mu \mathrm{g} / \mathrm{mL}$, at FCZ and VCZ, it presented minimal activity against $C$. auris planktonic cells (Table 1). APHB lipo was active against planktonic cells $(0.25-1.0 \mu \mathrm{g} / \mathrm{mL})$ and decreased metabolic activity of $C$. auris requiring much higher concentrations of the same antifungal $(16 \mu \mathrm{g} / \mathrm{mL})$. APHB was more effective, requiring $4 \mu \mathrm{g} / \mathrm{mL}$ to be more active against biofilm. The MCA was the most active ECHs, with $<0.5 \mu \mathrm{g} / \mathrm{mL}$ to inhibit planktonic cells, compared to 2-32 $\mu \mathrm{g} / \mathrm{mL}$ for CPA. However, these two antifungal agents, MCA and CPA were ineffective against biofilm ( $>32 \mu \mathrm{g} / \mathrm{mL}$ ). The CXA presented higher activity, with $<0.02 \%$ to inhibit, in fact, planktonic and sessile cells (Table 1). Among the antifungals analyzed, CPA was inactive against C. auris biofilm, which is noteworthy, because this antifungal agent is effective against Candida spp. biofilms. These factors may influence the virulence and survival of $C$. auris, and contribute to the outbreaks reported in hospital environments.

Susceptibility studies conducted by Chowdhary et al. ${ }^{12}$ demonstrated that $C$. auris presented high MIC to FCZ (CIM90 > $64 \mu \mathrm{g} / \mathrm{mL}), \mathrm{VCZ}$, APHB $(2 \mu \mathrm{g} / \mathrm{mL})$ and the ECHs (Table 1). The CXA presented efficacy as disinfectant in the prevention and control of infection

Table 1: Susceptibility of clinical isolates of $C$. auris to in vitro treatment with various antifungal agents.

\begin{tabular}{|c|c|c|c|c|c|c|c|c|c|c|c|c|}
\hline \multicolumn{13}{|c|}{ MIC $(\mu \mathrm{g} / \mathrm{mL})$ and $\mathrm{MIC}_{90}$} \\
\hline FLZ & VCZ & ICZ & PSZ & ECHs & MCA & CPA & ADA & APHB lipo & APHB & FCA & CXA & References \\
\hline$>32$ & & & & & $<0.5$ & $2-32$ & & $0.25-1.0$ e $16^{*}$ & 4 & & $<0.02 \%$ & 13 \\
\hline$>64$ & 2 & & & & & & & & 2 & & & 12 \\
\hline \multirow[t]{3}{*}{$>256$} & 2 & RV & RV & RV & & & & & $\mathrm{RV}$ & & & 6 \\
\hline & & $\geq 2$ & & & & & & & & & & 15 \\
\hline & & & & & & & & & $>1$ & & & 27 \\
\hline$\geq 32$ & $\geq 2$ & & & $\geq 8$ & & & & & $\geq 2$ & $\geq 128$ & & 26 \\
\hline$\geq 32$ & $\geq 2$ & & & & & & $\geq 2$ & & $\geq 2$ & & & 9 \\
\hline
\end{tabular}

MIC (minimum inhibitory concentration); $\mathrm{MIC}_{90}$ (minimum inhibitory concentration capable of inhibiting $90 \%$ of isolates); $\mathrm{FCZ}$ (fluconazole); VCZ (voriconazole); ICZ (itraconazole); PSZ (posaconazole); ECHs (echinocandins); MCA (micafungin); CPA (caspofungin); ADA (anidulafungin); APHB lipo (amphotericin B liposomal); APHB (amphotericin B); FCA (flucytosine); CXA (chlorhexidine). *MIC related to biofilm cells. 
by $C$. auris, through hand hygiene, oral gargling and skin disinfection, as well as topical use of nystatin and terbinafine ${ }^{12}$.

Gaitán et al. ${ }^{6}$ observed that $C$. auris is resistant to $\mathrm{FCZ}$ and $\mathrm{VCZ}$ and has MIC variable to $\mathrm{ECHs}$ and $\mathrm{APHB}$. The isolates were resistant to $\mathrm{FCZ}$ (MIC > $256 \mu \mathrm{g} / \mathrm{mL}$ ), VCZ (MIC $2 \mu \mathrm{g} / \mathrm{mL}$ ) and to PSZ, ICZ, ECHs and APHB (Table 1$)^{6}$.

Kumar et al. ${ }^{15}$, observed that $C$. auris was ICZ-resistant (MIC $\geq 2 \mu \mathrm{g} / \mathrm{mL}$ ), indicating expressive virulence factors, including phospholipase, proteinase and hemolysin activity (Table 1 ).

Calvo et al. ${ }^{27}$, between March 2012 and July 2013, analyzed C. auris in 18 patients with candidemia, from a medical center in Maracaibo, 13 pediatric patients, with men age of 26 days and 5 adults. All had received antibiotics and invasive procedures, at the onset of treatment with antifungal drugs the catheter was removed. Patients' survival was of up to 30 days. Through AFLP fingerprinting the isolates were genotyped and suggested a clonal outbreak. The isolates were azole-resistant, susceptible to anidulafungin (ADA) and MIC $>1 \mu \mathrm{g} / \mathrm{ml}$ for APHB (Table 1$)^{27}$.

Lockhart et al. ${ }^{26}$, found MICs to $C$. auris with the following antifungal drugs: $F C Z(\geq 32 \mu \mathrm{g} / \mathrm{mL}), \mathrm{VCZ}$ $(\geq 2 \mu \mathrm{g} / \mathrm{mL}$ ), ECHs ( $\geq 8 \mu \mathrm{g} / \mathrm{mL}$ ), flucytosine (FCA) $(\geq 128 \mu \mathrm{g} / \mathrm{mL})$ and APHB $(\geq 2 \mu \mathrm{g} / \mathrm{mL})$, the values found, in the present study, were similar to the MICs published by ANVISA (2017), FCZ ( $\geq 32 \mu \mathrm{g} / \mathrm{mL})$, $\operatorname{VCZ}(\geq 2 \mu \mathrm{g} / \mathrm{mL})$, APHB $(\geq 2 \mu \mathrm{g} / \mathrm{mL})$, and ECHs (ADA $\geq 2 \mu \mathrm{g} / \mathrm{mL}$ ) (Table 1) .

In the United Kingdom, a hospital verified the utility of chlorine in hydrogen peroxide products and the in vitro activity of chlorhexidine against $C$. auris. The use of chlorhexidine disposable table cloths for surface cleaning and hand hygiene as well as chlorhexidine alcohol prior to intravenous catheter manipulation resulted in a $95 \%$ decrease in the incidence of BSIs by C. auris after application of these measures ${ }^{8}$.

Thus, it is observed that $C$. auris presents in general a very variable susceptibility to different classes of antifungal and other antimicrobial agents analyzed, with resistance in the majority of cases, which makes treatment difficult. For each new isolate of $C$. auris, it is recommended the use of antifungigram tests to determine MIC, as this should help to select the most appropriate medicine and accelerate the patient's clinical response, who is at serious risk of death when affected by this yeast.

\section{CONCLUSION}

C. auris is a multidrug-resistant, pathogenic yeast that can be a source of healthcare-associated infections in hospitals. It has a high potential for horizontal nosocomial transmission. In order for health services to be able to fight these infections, implementing control and prevention measures, it is necessary for laboratories to accurately identify C. auris. Thus, the competent authorities will be notified of possible outbreaks, and may adopt appropriate measures, controlling the epidemic.

Routine laboratory methods are insufficient for detection of $C$. auris, which may be erroneously identified as other species. The correct identification of the yeast requires sophisticated molecular methods for proper confirmation at the species level. In suspect cases the isolate should be sent to a reference laboratory capable of identifying the $C$. auris species.

Its epidemiology is variable and $C$. auris has no specific location, being widely found around the world. In Brazil, it is still a controversial issue, because although there are no reports of infection by the species in the country, its incidence is not reliable due to the obstacles found in its identification and diagnosis, a fact that compromises the effective fight against the spread of the yeast.

Chlorhexidine should be seen as a possible strategic solution in preventing $C$. auris infections, serving as a skin disinfectant. Its treatment is still limited, due to the antifungal resistance, an intrinsic species' trait. Currently, there is no evidence that point to an effective standard treatment. This fact, coupled with the high association with mortality, makes $C$. auris an emerging global threat.

Finally, $C$. auris is a species currently responsible for many outbreaks, which has been generating public health alert in Brazil and other countries. Surveillance and Monitoring Agencies in Health Services have issued notes of numerous reports of cases of infections by this yeast, which hardly responds to conventional treatment. Thus, further research should be developed to elucidate the risk factors as well as the transmission mechanism, allowing accurate and effective guidelines for the population to take the necessary actions and precautions to avoid the spread of this pathogen.

\section{Conflict of interest}

The authors declare no conflicts of interest. 


\section{REFERENCES}

1. Barbedo LS, Sgarbi DB. Candidíase. DST - J Bras Doenças Sex Transm. 2010;22(1):22-38.

2. Giolo MP, Svidzinski TI. Fisiopatogenia, epidemiologia e diagnóstico laboratorial da candidemia. J Bras Patol Med Lab. 2010;46(3):225-34. http:// dx.doi.org/10.1590/S167624442010000300009.

3. Colombo AL, Guimarães T. Epidemiologia das infecções hematogênicas por Candida spp. Rev Soc Bras Med Trop. 2003;36(5):599-07. PMid:14576875. http://dx.doi.org/10.1590/S003786822003000500010 .

4. Ben-Ami R, Berman J, Novikov A, Bash E, Shachor-Meyouhas Y, Zakin S, et al. Multidrug-Resistant Candida haemulonii and C. auris, Tel Aviv, Israel. Emerg Infect Dis. 2017;23(1):195-03. PMid:28098529.

5. McCarty TP, Pappas PG. Invasive Candidiasis. Infect Dis Clin North Am. 2016;30(1):103-24. PMid:26739610. http://dx.doi.org/10.1016/j. idc.2015.10.013.

6. Gaitán ACR, Moret A, López Hontangas JL, Molina JM, Aleixandre López Al, Cabezas $\mathrm{AH}$, et al. Nosocomial fungemia by Candida auris: First four reported cases in continental Europe. Rev Iberoam Micol. 2017;34(1):237. PMid:28131716. http://dx.doi. org/10.1016/j.riam.2016.11.002.

7. Kaaniche FM, Allela R, Cherif S, Algia NB. Invasive candidiasis in critically ill patients. Curr Anaesth Crit Care. 2016;11:1-5.

8. European Centre for Disease Prevention and Control (ECDC). Candida auris in healthcare settings - Europe. Stockholm: ECDC; 2016 [cited 2017 Sep 11]. Available from: http://ecdc.europa.eu/en/publications/ Publications/Candida-in-healthcaresettings_19-Dec-2016.pdf.

9. Agência Nacional de Vigilância Sanitária (ANVISA). Comunicado de Risco $n^{\circ}$ 01/2017 - GVIMS/ GGTES/ANVISA: relatos de surtos de Candida auris em serviços de saúde da América Latina. Gerência de Vigilância e Monitoramento em Serviços de Saúde, Gerência Geral de Tecnologia em Serviços de Saúde e Agência Nacional de
Vigilância Sanitária. Brasília: ANVISA; 2017 [cited 2017 Sep 11]. Available from: http://portal.anvisa.gov.br/ documents/33852/458700/Comunica do+de+Risco+n\%C2\%BA+01+2017 +GVIMS-GGTES-Anvisa/1d23b2005640-4aa3-a8e8-5239c8d2e000.

10. Choi HI, An J, Hwang JJ, Moon SY, Son JS. Otomastoiditis caused by Candida auris: Case report and literature review. Mycoses. 2017;60(8):488-92. PMid:28378904. http://dx.doi.org/10.1111/myc.12617.

11. Carenzi LR, Silveira F, Faidiga GB, Lago T, Yassuda CC, Massuda $E T$, et al. Mastoidite fúngica em paciente com SIDA: relato de caso. Arq Int Otorrinolaringol. 2011;15:245-8.

12. Chowdhary A, Voss A, Meis JF. Multidrug-resistant Candida auris: 'new kid on the block' in hospitalassociated infections? J Hosp Infect. 2016;94(3):209-12. PMid:27634564. http://dx.doi.org/10.1016/j. jhin.2016.08.004.

13. Sherry L, Ramage G, Kean R, Borman A, Johnson EM, Richardson $M D$, et al. Biofilm-forming capability of highly virulent, multidrug-resistant Candida auris. Emerg Infect Dis. 2017;23(2):328-31. PMid:28098553. http://dx.doi.org/10.3201/ eid2302.161320.

14. Morales-López SE, Parra-Giraldo CM, Ceballos-Garzón A, Martínez HP, Rodríguez GJ, Álvarez-Moreno $\mathrm{CA}$, et al. Invasive Infections with Multidrug-Resistant Yeast Candida auris, Colombia. Emerg Infect Dis. 2017;23(1):162-4. PMid:27983941. http://dx.doi.org/10.3201/ eid2301.161497.

15. Kumar A, Sachu A, Mohan K, Vinod V, Dinesh K, Karim S. Simple low cost differentiation of Candida auris from Candida haemulonii complex using CHROMagar Candida medium supplemented with Pal's medium. Rev Iberoam Micol. 2017;34(2):10911. PMid:28392225. http://dx.doi. org/10.1016/j.riam.2016.11.004.

16. Satoh $\mathrm{K}$, Makimura K, Hasumi $\mathrm{Y}$, Nishiyama Y, Uchida K, Yamaguchi H. Candida auris sp. nov., a novel ascomycetous yeast isolated from the external ear canal of an inpatient in a Japanese hospital. Microbiol Immunol. 2009;53(1):41-4. PMid:19161556. http://dx.doi.org/10.1111/j.13480421.2008.00083.x.
17. Prakash A, Sharma C, Singh A, Kumar Singh $P$, Kumar A, Hagen $F$, et al. Evidence of genotypic diversity among Candida auris isolates by multilocus sequence typing, matrix-assisted laser desorption ionization time-of-flight mass spectrometry and amplified fragment length polymorphism. Clin Microbiol Infect. 2016;22(3):277. e1-9. PMid:26548511. http://dx.doi. org/10.1016/j.cmi.2015.10.022.

18. Sharma C, Kumar N, Pandey R, Meis $\mathrm{JF}$, Chowdhary A. Whole genome sequencing of emerging multidrug resistant Candida auris isolates in India demonstrates low genetic variation. New Microbes New Infect. 2016;13:77-82. PMid:27617098. http://dx.doi.org/10.1016/j. nmni.2016.07.003.

19. Emara M, Ahmad S, Khan Z, Joseph L, Al-Obaid I, Purohit P, et al. Candida auris candidemia in Kuwait, 2014. Emerg Infect Dis. 2015;21(6):10912. PMid:25989098. http://dx.doi. org/10.3201/eid2106.150270.

20. Vallabhaneni S, Kallen A, Tsay S, Chow N, Welsh R, Kerins J, et al. Investigation of the First Seven Reported Cases of Candida auris, a Globally Emerging Invasive, MultidrugResistant Fungus-United States, May 2013-August 2016. Am J Transplant. 2017;17(1):296-9. PMid:28029734. http://dx.doi.org/10.1111/ajt.14121.

21. Pan American Health Organization (PAHO), World Health Organization (WHO). Epidemiological alert: Candida auris outbreaks in health care services. Washington: PAHO: WHO 2016 [cited 2017 Sep 11]. Available from: http://www.paho.org/hq/index. php?option $=$ com_docman\&task $=$ doc view\&ltemid=270\&gid $=36354$ \&lang $=\bar{e}$ $n \% 3 \mathrm{E}$.

22. Rudramurthy SM, Chakrabarti A, Paul RA, Sood P, Kaur H, Capoor MR, et al. Candida auris candidaemia in Indian ICUs: analysis of risk factors. J Antimicrob Chemother. 2017;72(6):1794-801. PMid:28333181. http://dx.doi. org/10.1093/jac/dkx034.

23. Chowdhary A, Sharma C, Duggal S, Agarwal K, Prakash A, Singh PK, et al. New clonal strain of Candida auris, Delhi, India. Emerg Infect Dis. 2013;19(10):1670-3. PMid:24048006. http://dx.doi.org/10.3201/ eid1910.130393. 
24. Magobo RE, Corcoran C, Seetharam S, Govender NP. Candida auris-associated candidemia, South Africa. Emerg Infect Dis. 2014;20(7):1250-1. PMid:24963796. http://dx.doi. org/10.3201/eid2007.131765.

25. Schelenz S, Hagen F, Rhodes JL, Abdolrasouli A, Chowdhary A, Hall $A$, et al. First hospital outbreak of the globally emerging Candida auris in a European hospital. Antimicrob Resist Infect Control. [eCollection 2016]. 2016;5:35.
26. Lockhart SR, Etienne KA,

Vallabhaneni S, Farooqi J, Chowdhary

A, Govender NP, et al. Simultaneous

Emergence of Multidrug-Resistant

Candida auris on 3 Continents

Confirmed by Whole-Genome

Sequencing and Epidemiological

Analyses. Clin Infect Dis.

2017;64(2):134-40. PMid:27988485.

http://dx.doi.org/10.1093/cid/ciw691.

27. Calvo B, Melo AS, Perozo-Mena A, Hernandez M, Francisco EC, Hagen F, et al. First report of Candida auris in America: clinical and microbiological aspects of 18 episodes of candidemia.
J Infect. 2016;73(4):369-74.

PMid:27452195. http://dx.doi. org/10.1016/j.jinf.2016.07.008.

28. Larkin E, Hager C, Chandra J, Mukherjee PK, Retuerto M, Salem I, et al. The Emerging Pathogen Candida auris: growth phenotype, virulence factors, activity of antifungals, and effect of scy-078, a novel glucan synthesis inhibitor, on growth morphology and biofilm formation. Antimicrob Agents Chemother. 2017;61(5):e0239616. PMid:28223375. http://dx.doi. org/10.1128/AAC.02396-16. 